\title{
ÉLECTRICITÉ
}

\section{L'électrification des chemins de fer en Suisse}

\author{
par G. Kolovitch, Ingénieur I. E. G.
}

\section{Considérations générales}

Parmi les pionniers de l'électrification des moyens de transport, les Suisses ont eu surtout le mérite d'avoir développé un effort considérable et ont eu, comme il était juste, la satisfaction d'avoir obtenu des résultats qui leur font honneur.

En matière de réalisation des projets délectrification des chemins de fer, plusieurs tentatives ont été faites. Le problème est complexe et plusieurs solutions peuvent sembler la " meilleure ». Toutes ont leur raison d'être, mais elles sont aussi indistinctement passibles de critiques. Le meilleure résultat s'obtiendra avec le système dont on approfondira le mieux les caractéristiques et qui deviendra par la suite, grâce aux enseignements de l'exploitation, le plus perfectionné. La solution heureuse ne devra pas être pressentie ni devinée, mais créée par des efforts techniques efficaces. Les techniciens Suisses ont choisi une méthode, ils ont créé leur Ecole. Ils ont rendu presque parfaite, l'exploitation en traction électrique par le monophasé.

Le problème de la traction électrique est, par excellence, un de ceux, à la fois très complexes et vitaux pour une région, le plus souvent, et quelquefois même, pour une nation entière. C'est le cas typique pour la Suisse, ainsi que pour l'Italie.

Ici le problème a été aussi bien d'ordre social que d'ordre national.

Développer l'industrie des chutes d'eau revenait à donner un essor puissant à une industrie nationale, avec toutes les conséquences de bien-être qui en découlent. Emancipation du Pays de l'étranger, arrêt d'une fuite importante des capitaux par la diminution du chiffre "importations" avec une augmentation subséquente du chiffre "exportations ", puisque la Suisse vend à des pays voisins une certaine quantité, en fait assez élevée, d'énergie électrique.

La diminution importante de la consommation de la houille noire a permis, avec les capitaux qui étaient affectés à son acquisition, de développer l'industrie nationale hydro-électrique et, avec elle, celle des constructions électriques, qui notamment, dans le domaine du matériel de traction a atteint un degré de perfectionnement tel que l'exploitation corrélative s'exécute dans le monde entier, le matériel suisse étant agrée par de nombreux pays où l'industrie électromécanique est très développée.

Ainsi, ressort très clairement tout l'intérêt de l'ellectrification des chemins de fer Suisses, lorsqu'on envisage ce problème sous l'angle de l'économie nationale.

Les conséquences de l'électrification d'une région ont une portée le plus souvent remarquable. Il en résulte une amélioration économique considérable. Une infinité de facteurs interviennent en sa faveur. Ils sont souvent imperceptibles, avant la mise en ceuvre d'une telle exploitation; ce n'est que plus tard, qu'une série croissante d'agréables constatations met en lumière les avantages de l'exploitation d'un réseau électrifié.

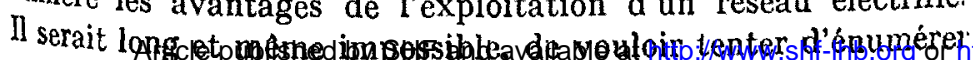

ces avantages. Aussi, nous bornerons-nous à ne signaler que les plus saillants.

L'entretien du matériel roulant est énormément facilité. On n'a pas eu de peine à constater que sa durée de service, qui en résulte, est assez sensiblement augmentée.

Que dire des conséquences sociales ? Elles sont tout simplement remarquables ! La vie de tout le personnel a totalement changé, On a tellement été habitué à la figure symbolique du cheminot, pour qui, par la force des choses, la propreté extérieure semblait quelque peu incompatible, que l'on se refuse à le reconnaître lorsqu il fait partie du personnel d'un réseau électrifié.

Et pourtant, là encore, le résultat n'est pas aussi frappant que lorsque l'on envisage le changement de vie des mécaniciens et des chauffeurs. Ils ont vu leur dur métier complètement transformé. A la vie épuisante des quelques heures de service qu'ils avaient autrefois à effectuer, s'est substituée une autre vie qui n'est guère plus pénible que celle d'un employé de bureau. L'effort considérable, la résistance physique, les dangers qui constituaient le lot de cette classe de travailleurs ont totalement disparu, et c'est là un grand bien. Nous avons pu constater, malgré cela, que nombre d'entre eux, tout en reconnaissant les bienfaits de la transformation, regrettent un peu le travail auquel ils s'étaient endurcis et qui les surclassait intellectuellement. Leur tâche étant actuellement si simplifiée, ils sentent leur valeur sociale diminuer.

De ce fait, une crainte les saisit de se sentir devenir moins indispensables. Lorsque pour arriver à manier une motrice à vapeur, il fallait plusieurs années d'initiation, avant de pouvoir se glorifier du titre envié de mécanicien, aujourd'hui on peut former un bon électromachiniste en un temps très court. Il ne faut pas mésestimer ces regrets très humains.

Dans une motrice à vapeur, le mécanicien est l'âme agissante. De lui, de sa valeur, tout dépend. Certes, c'est là un point qui n'est pas à négliger et qui explique l'attachement que ces déraciné portent encore, malgré tout, à leurs "fumeuses ".

Il y a aussi la crainte du nivellement par en bas, de la banalisation des valeurs, génératrice de chômage.

\section{Considérations d'ordre technique}

Le choix de la forme de courant à employer pour les réseaux à électrifier s'est porté sur le monophasé. Les techniciens suisses ont-ils, dès l'abord, entrevu les difficultés innombrables qui apparaissent, en choisissant le monophasé, et en sy limitant strictement? Il nous est difficile de donner une réponse bien nette. Nous pouvons, toutefois, affirmer que les résultats obtenus aujourd'hui, avec le système choisi, sont des plus satisfaisants. Quel aurait été actuellement le résultat s'ils avaient opté pour un autre système? C'est ce qui serait très intéressant,

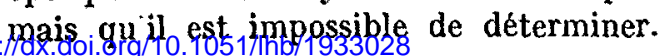


Nous exposerons maintenant, trè's brièvement, quelques considérations d'ordre général touchant le choix du type de courant à employer, pour lélectrification d'un réseau de chemins de fer.

\section{Caractéristiques des moteurs}

Les moteurs à courant continu semblent etre les plus aptes a assurer un servore de raclion. La sonplesse de ces moteurs est sans aucun doute la meilleure: pour un réseau accidenté, le moteur sera appelé à fonctionncr fréquemment à des régimes de vitesses el de charges très différentes, lant à cause du profil de la ligne, que du fail de la variation du trafic, le moteur à courant continu se prètera micux à ces exigences très variables en maintenant son rendement dans les meilleures conditions possibles. Il reste donc actuellement le préféré pour les exploilations à régimes journaliers variables. (réseaux de Iramways métropolitains et nombre de lignes interurbaines), et chaqu: fois que d'autres considérations interviennent, savoir : transport d'énergie, ligne de prise de courant, sous-station d'alimentation, solution thermoélectrique. C'est ainsi que pour les automolrices Diesel-électriques, le courant continu est seul employé.

Si le collecleur donne à ce moleur des propriétés aussi remarquables, il nicst pas non plus exempt de critiques.

La commulation constilus un problème très délicat. Des difficullés apparaissenl, avec le moteur à courant continu, qui surtout innitent la tension d'exploitation, ce qui n'est pas sans imporlance, conme nous le verrons par la suite.

Entre les divers moleurs à courant alternatif, on concoit facilement que le choix doive se porter sur les moteurs polyphasés. L cur fonclionnement est le plus convenable et surtout leur couple plus régulier et plus continu. N'envisageons que le hanal moteur asynchrone triphasé. Il a déjà, à son aclif, un crlain nombre d'avantages, qui ne sonl pas à négliger. Simplicité de construction, puissance massique très élevée, possibilité d'alimenlation à haute lension jusqu'à 20 ou $25 \mathrm{kv}$.

Mais combien il est moins souple que son frère à courant continu, quelle difficulté pour obtenir avac lui un peu de souplesse de réglage et, lorsqu'on y parvient, à quels frais!

D'autre part, pour lui assurer un fonctionnement normal et lúi éviter un échauffement exagéré, il paraît plus prudent de lalimenter par une sourcs à fréquence peu élevée.

La moteur monophasé reste le plus désavantagé, car ll porte avec lui la tare des autres, généralement beaucoup plus accentuée. Son circuil magnétique est mal utilisé ; il en résulte une puissance massique assez réduite. Sa somplesse est inférieure à celle dis deux autres. Mais, comme nous le sous-entendons, il s'agit là du moteur asynchrone monophasé ordinaire. Or, avec. les perfectionnements apportés aux moleurs électriques à collecteur, alimentés par du courant allernatif, leur exploitation est dzvenue réellement satisfaisante. Je collecleur leur confère en partie celle propriété si précieuse des moteurs à courant continu: la souplesse de réglage aux diverses charges. Dès lors, la valeur des moteurs à courant alternatif est plus marquée el plus active la concurrence qu'ils opposent aux moteurs à courants continus.

La discussion du choix de la nature du courant à cmployer pour l'électrification d'un réseau de traction doit désormais se porter sur un terrain tout autre, savoir : facilité de réalisation et d'exploitation, ainsi que sur le prix d'établissement et sur le rendement en exploitation du dit réseau.

\section{Distribution de l'énergie et alimentation des locomoteurs}

Sous ce tilre, nous nous proposons de donner quelques ind calions d ordre très général, plus désireux de poser les problèmes que de les résoudre.

Lignes de prese de courant. - L élablissoment des lignes d'amenée du courant offre une infinite de difficultes d'ordre lechnique, d'une gravité beaucoup phus grande que celle pouvant apparaître à première vuc. On peul, en offet, affirmer actuellement que le systeme de traction par courant triphasé, doil la plupart du tempes son infériorité, par rapport aux autres systèmes, uniquement aux complications qui résultent de ses lignes de contact. Son exécution est des plus délicales, en parlieuhier aux croisements, bifurcations el surtout ì l'entré ders gares, el devient presque impossible lorsque l'inportance of th nombre de ces dernièrcs dépasscnt une certaine limile.

C'est en Italic, presque exclusivement, que les tentalives européennes de traction avec le courant triphasé ont été pratiquées et non sans de louables résultats. Des lignes de lraction nombreuses pour courant triphasé fonctionnent, d l'unc tout dernièrement équipéc à la fréquence normale de 50 périodes.

Ce choix devrait constituer un facteur essentiellement favo. rable pour le développement de ce genre de Iraction, car il offe un avantage des plus importants, celui de permetle dinkrconnecter les réscaux d'énergie électrique ferroviaires à ceux des lignes de transport deslinées aux diverses industries de la région.

'Toutefois, on peut affirmer que, pour l'instant, tout au moins, le système de traction par courant triphasé cst en veilleuse. et la meilleurt preuve nous en est donnéc par le fail que ces promoteurs italiens ne l'envisagent plus pour les nouvelles lignes, qu'ils onl à électrifier.

Les difficultés yui subsistent pour l'alimentation des locomoteurs, fonclionnant au moyen du courant continu, sont loin d'ètre sans importance. Avec ce système, qui pourtant est lit plus apte au service de traction, le problème de la captation du courant représente encore un point tellement faible, quill permet au syslème concurrent, le monophasé, de se situer, etr définitive, d'une façon presçue aussi avantageuse. Le fail qu la tension d'exploitation est actuellement limitée en praligut à $3.000 \mathrm{~V}$, et que, mème celle d'avenir envisagée ne dépasset pas, au grand maximum, $6.000 \mathrm{~V}$., impose de transporter l'éne:gie sous une inlensılé très élevéc, et d'en supporter tous los inconvénients qui en découlent. Voir : pertes en lignes trós importanles ainsi que nécessité d'un nombre de sous-stationt d'alimentation très grand.

Ce qui précède suffil à montrer loule l'imporlance du problème, car avec les sous-stations ainsi mullipliées, les frais d’et blissement s'accroissent et le rendement global diminue.

Tel est pour le système de traction à courant continu lé griét le plus important. L'importance n'en est pas négligeable. Malgri cela, et du fail des qualités lechniques des moleurs à courarit conlinu particulièrement favorables pour le service de tractiot ce système est encore très employé. C'est surtout en lirank où il est presque exclusivement adopté, qu'il est le plus en favelin On le maintient el on le préconise pour loule électrificatiat actuelle en projet. En Italie, où s'étail créé un vaste laboric

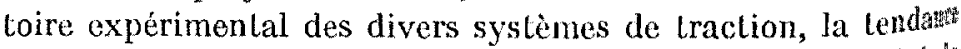
définilive semble se porter presque exclusivement du cótë du courant conlinu. L'électrification de la presque lolalilé des not velles lignes s'effectuera, en effet, avec du courant continu. Lot perfectionnements, tout dernièrement apporlés aux redresse 
a rapeur, de mercure, les qualites qui les rendent aptes aujourdhut a assurer des services durs ol continus. sous des charges très édeveses, constiluent un argument d'importance très apprécalible colaveur du courant continu, le rendement de ces appareils statiques est manifestoment supérieur à celui des groupes convelisscurs qui les ont precédes. Laublomatisine des sous-

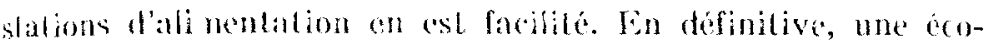

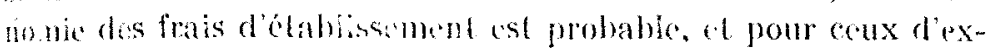
ploblation, a roduction quil ast possible doblenar est tres rumarquabie.

Dans la das du comtant continu d devant lobligation davoir a transporter une intensile tres focte, on a essaye de capter le comant par troisicine rail el sabols frotteurs. Ie probleme dannentalion du tracteur reste loujours assez delical car, a diflerentes eporques, ol sur diverses lignes, on a, devant choisir

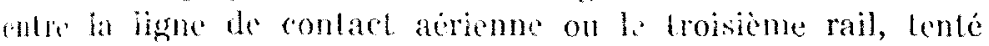
Fexperiene simbance de ces deux mod s de prise de courant

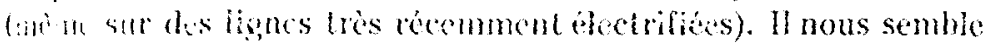

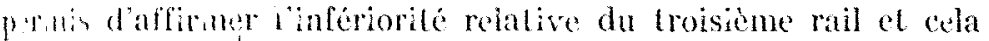
pual trourer une confirmation dans le fail que la plupart des risalle recommont chelrilies so sont armetes at uns ligne de contact aerienne.

1. Troisiome rati presente des inconvenients plus nombrux : diffinthi d"isolenent, danger continu le long de la voie, fuiles par cotranls ragabonds facilités par le mauvais lemps : pluie (1) 11 ige, inconventents de la coupure aux passages à niveau it surbut dins ies gares pour les aiguillages. Pendant les époques d. frodd rigonrix très somvent, contacts defectueux provoqués par how.

La tracion par courant monophasc est, par principe, au point d. ver genchal, asser desavantagée. Puissance massique des molenrs encore un pill plus faible que pour les autres solutions. Cartchistigues dil couple moteur aussi moins favorables. On pul, presque systematiquement, affirmer que lo rendement du notur la lraction monophasé pour des conditions identiques de nardwe, est le plus faible. Ce genre de courant n'étant plus ntilste comanment pour d'autres industries, il en résulte que lechange de lonerpio avec les autres réseaux par l'interconnexion asl impossible.

Was à crite de cos inferiorités rolatives aux autres moteurs, l. monoteur monophasé a pour hij des qualites fort appréciables. dont les diux principales : possshibite dalimentation a hate

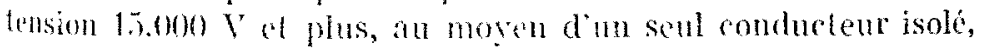
suffirail à en afïmer la valcur.

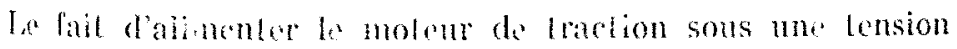
ansi dever pemel dispacer les sous-stations de transfor-

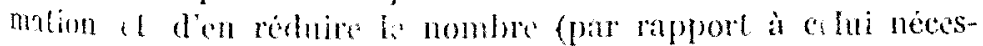
sate ave fo courant continu dans des condilions idintiques),

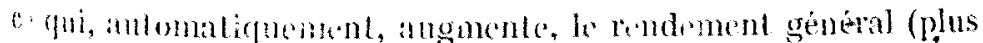

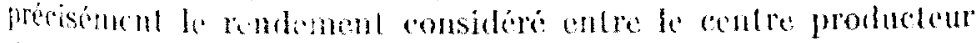

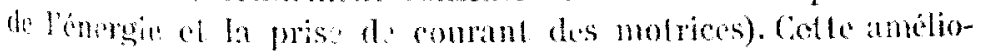
ration est tres appreciable.

baule part, Ia circulation du courant, entre les sous-stations datimentation soffectue sous intonsite baacoup plus faible, "l d'aulant plas, que la lension de service est plus elevé, ce qui 'ntrain: un: economie substantielte des frais d'installation.

Lorsqutune région assez importante par le nombre de voies ferées on par lear trafic doil clre enterement dectrifies, dle poura rationndlement axiger la mise sur pied de centres producleurs d'énergic bluctrique, uniquement destinés à l'usage resservics d: lraclion, val linpurtance de la puissane moyenne de ces services.
Dans ce cas particulier, ces centrales électriques peuvent ètre construites suivant les données que le service de traction inpose, du moment que l'on est sûr que tout 'énergie produite sera consommée dans les conditions voulues. La possibilité d'échange de l'énergie avec d'autres réseaux perd un peu de son intérêt. Co cas est typique en Suisse. Les $($. . IF. F., ayant électrifué la presque lolalité des réseaux, réclamaient une quanlité d'énergie électrique trés élevé. Hs ont done préféré, et c’uat logique, produire cux-mèmes l'énergie qui leur est nécessaire, done dans les conditions voulues (nature du courant et fréquence).

C'est ainsi que les diverses usines hydro-électriques des C.F.F. sont interconneclées par un réseau de transport d'énergie à $65.000 \mathrm{~V}$., formant une boucle centrale de laquelle l'énergie électrique rayonne au moven de lignes a $15.000 \mathrm{~V}$. et alimente la voie ferré. Lénergie d. ces centrales, exclusivement deslinée à la traction, est directemont produile à $16,2 / 3$ périodes. Une grandr quantite de cette energie provient des usines de la valiée du lihone, utilisant au moyen de Iravaux de génie civil d'un intérêt technique de premier ordro, l'énergie hydraulique de la chaînt des Alpes Valaisanes et Bernoises. Une des plus belles unilés est actucllement lusine de Vernayaz. Da Vevey, cette boucle principale traverse te plateau cicntral et réunit entre elles les usines situées au pied du Jura, se refermant par les Préalpes, en suivan les vallées dis aflluents du lac des Qualre Cantons.

Le choix du courant monophasé n'est donc pas aussi désavantageux Iorsque l'exploitant dos voies ferrées électrifiées produit lui-même l'énergie nécessaire. Car, ce ne serail un inconvénient qu'au cas où celle énergie devrait être livrée sous forme massive par des producteurs d'énergie destinée à d'autres industries et qui est aujourd'hui uniformément transmise sous forme de courant triphasé. Dans ces conditions, lavantage que l'on tire, tant au point de vue coût d'installation, que frais d'exploitation du monophasé, par la réduction clans une proportion tries importante, du nombre des sous-stations dalimentation (par comparaison avec le courant continu) serait déja largement annihilé par l'augmentation du nombre des stations de Iransformation de courant triphasé en monophasé.

La puissance massique plus faible des moleurs monophasés n'est pas toujours un inconvénient surtout pour des lignes électrifiées avcc profils très accidentés. Dans des lignes de nontagnes un poids adhérent très élevé est nécessaire si l'on veut lirer tout le bénéfice possible de l'électrification qui est cn mesure de mettre à la disposition des locomoteurs toute la puissance qu'ils exigent.

D'autri part, lo moteur monophasé est réversible, le freinage écotrique ainsi que la récupéralion sont actuellement très aisés.

De loul chat on peul donc conclure que le courant monophasé présente aussi de sérieux avantages en traction d'où la justification de son largo emploi dans différentes régions, notamment en Suiss., en Autriche, en Allemagne, dans les régions Nordiques, enfin en Hongrie. Dans ce dernier pays, on procède actuellement activement a l'électrification de l'artère principale du réseau des chemins de fer. On y emploie l'énergit électrique sous forme d. courant monophasé pour le transport et l'alimentalion du locomoteur. Mais à la suite des magnifiques travaux de l'ingénieur $\mathrm{DF}$ Kaxoo, on essaye d'uliliser sur les locomotives son si ingénieux converlisseur de phases qui, avce un rendement excollent, permet, par Iransformation polymorphique sur le locomoleur meme, d'alimenter les moteurs de traction avec du courant polyphase en leur assurant, de ce fait, toute la souplesse voulue, ainsi qu'un couple régulier et continu: Propriétés si estimées pour le moteur à courant continu. La 
mise au point de ce nouveau système de DE KANDo, pourrail bien, si elle satisfait les espoirs des techniciens, constituer un terrible concurrent pour le courant continu.

\section{$*$}

La situation géographique de la Suisse et son relic tris accidenté imposent aux réseaux des C. F. F. un pare très varié. Pays interposé entre les nations les plus industriciles de l'Europe, il est transité par tout le trafic des échanges commerciaux qui s'effectuent entre ces différentes régions.

Les lignes de chemins de fer qui traversent la Suisse de lEst. à l'Ouest peuvent être prisque toutes considérécs comme des lignes de plaine où les déclivitès ne dépassent guère de 10 à $12 \%$ Par contre, les ligncs orientées suivant la direction Nord. Sud franchissent toutes des régions excessivement accidentés. qui s'y rencontrent ct qui lendent à se réduire aux quelques types répondant le mieux aux exigences du trafic.

L'expérience acquise dans la construction et dans l'explojlation de's locomotives fixe actuellement une donnée caractéristique générale du plus haut intérèt qui peut guider dans le choix des types de locomotives à adopter, nous voulons parler du poids adhérent par essieu noteur qui est évalué à 20 tonnes. D'aulre part, au poids adherent correspond pour chaque profil de la voie, un poids remarquable bien déterminé.

Il faut ainsi un polds adhérant de 22 à 27 tonnes pour remar. quer 100 tonnes sur une rampe de $26 \%$

Nous nous occuperons dans ce qui suit, de quelques types d'unités motrices qui se rencontrent le plus fréquemment, avant d'exposer d'une facon un peu plus générale, les caractéristiques des deux types de locomotives, actuellement en service

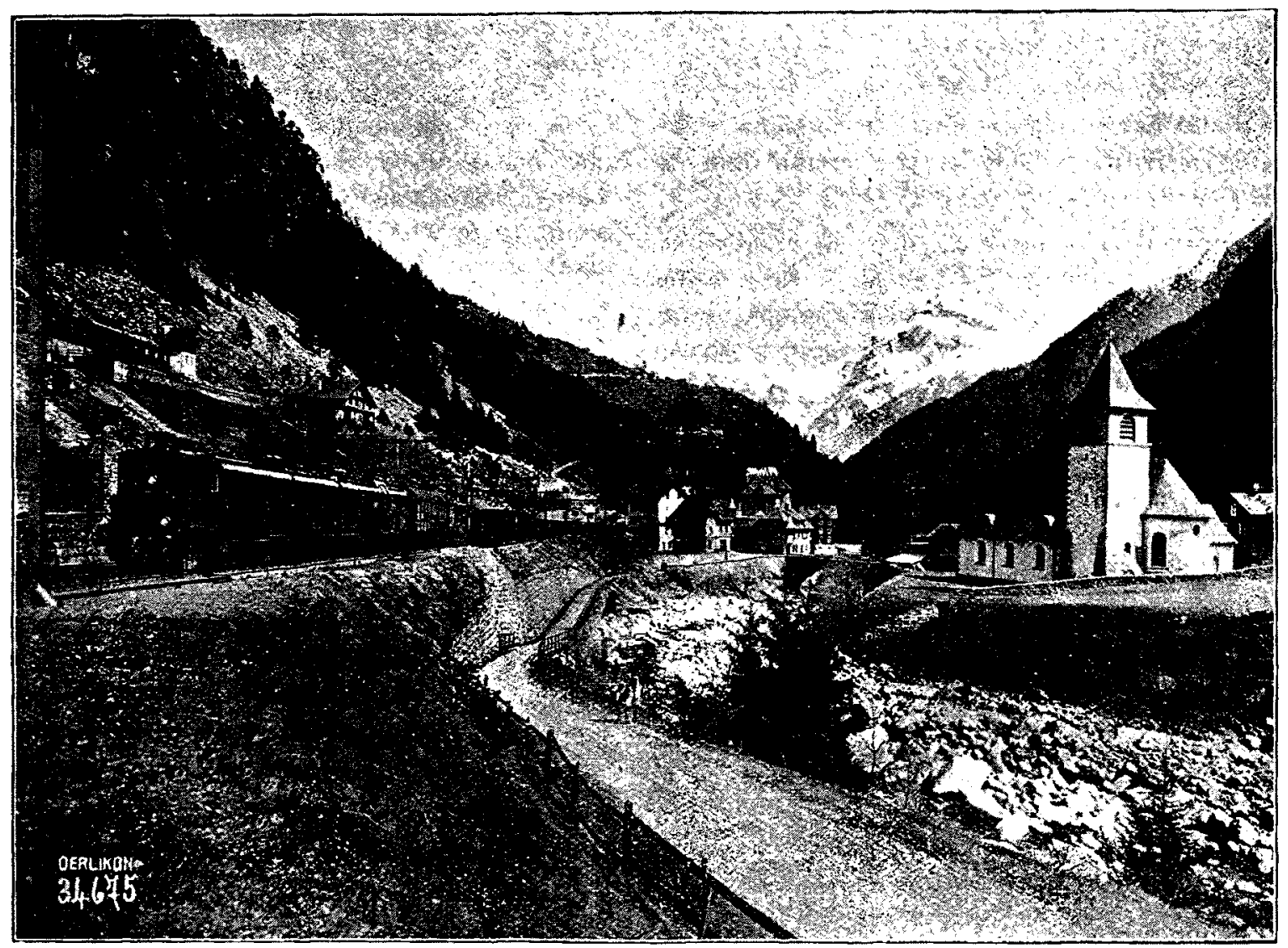

Fig. 1 - Ligne du Saint-Gothard

(Pholog' auhie Oerlikon)

ayant à gravir pendant des dizaines de kilometres d.s rampes de $27 \%$.

La composition des trains est, elle aussi, lrès variée. Des trains de 300 tonnes et 480 tonnis circulent normalement pour les échanges intérieurs ; mais, on note nombre de composiiions beaucoup plus importantes, de 600,700 et mème 1.400 tonnes provenant des plaines et destinées à franchir des lignes comme celle du Saint-Gothard (fig. 1).

Devant une telle variélé du trafic, il a été très difficile de fixer les caractéristiques des unités locomotrices afin d'en oblenr une utilisation rationnelle. La Suisse, pays ayant entripris l'électrification de sıs réseaux depuis les débuts, est divenu un véritable laboratoire d'essais et de recherches pour la traction qui, d'ailleurs, a donné les résultats les plus brillants, tant au point de vue organisation de l'exploitation qu'à colui de la construction du matéricl électrique de traction.

Ces considérations justifient la diversité des unités motrices sur la ligne du Saint-Gothard, ces deux derniers étant cerlajnement les plus intéressants.

\section{Série $A_{e} 3 / 5$ \\ Partie Mecanique}

Comme l'indique le nom de la série, il s'agit de locomotives à trois essicux moleurs et deux porteurs. Au point de vue force de traction, puissance el vilesse maximum les locomotives $A_{\mathrm{e}} 3 / 5$ ont à satisfaire aux condilion générales suivantes:

Remorque de trains de 480 tonnes sur rampe de $10 \%$ à la vilesse de $65 \mathrm{~km} / \mathrm{h}$., el sur rampe de $2 \%$ à la vitesse de $90 \mathrm{~km} / \mathrm{h}$ Lo maximum de vilesse cst fixè à $90 \mathrm{~km} / \mathrm{h}$.

J $\leftrightarrow$ s caractéristiques générales de ce type de locomolives sont lis suivantes :

Genre de courant ..............

Altur. monoph. $15 \mathrm{KV}$. et $162 / 3 \mathrm{pps}$ 
Poids de la locomotive en ordre de marche Poids adhérant ................. Empatlement fixt .............. Force de traction unihoraire à la jante. . Force de traction maximum au démarrage. puissanct unihoraire à la jante........ Puissance continue $\ldots \ldots \ldots \ldots \ldots \ldots$ Vilesse maximum $\ldots \ldots \ldots \ldots \ldots \ldots \ldots$ Nombre de moteurs . .............. Rapporl de réduction des engrenages.... Diamètre des roues motrices ........ Diamctre des roues porteuses ........
81,1 tonnes 55,5 -

$4.200 \mathrm{~mm}$.

$7.700 \mathrm{~kg}$. à $63 \mathrm{~km} / \mathrm{h}$.

$14.000 \mathrm{~kg}$.

$1.800 \mathrm{Ch}$. à $63 \mathrm{~km} / \mathrm{h}$.

$1.560 \mathrm{Ch}$. à $68 \mathrm{~km} / \mathrm{h}$.

$90 \mathrm{~km} / \mathrm{h}$.

6

$1 / 5$

$1610 \mathrm{~mm}$.

$930 \mathrm{~mm}$.

Chissis. - Il est constitué par des longerons extérieurs et. continus, qui ont $22 \mathrm{~mm}$. d'épaisseur ; ils sont entretoisés à intervalles de $1.900 \mathrm{~mm}$. par les traverses de tête, par des supports transversaux au-dessus des essicux-porteurs, ainsi qu'en avant el en arrière des essieux-moteurs.

Les longerons extérieurs sont exigés à cause du mode de transmission qui, pour ces locomotives, est la commande individuelle des ussieux, système Westinghouse.

De fortes entretoises relient les cornières inférieures des longerons sous les boîtes d'essieux-moteurs.

Les carcasses des moteurs reposent sur des traverses boulonnées à des supports fixés aux entretoises du châssis. Après alevement des traverses, chaque paire de moteurs avec l'essieumoteur correspondant se démontent facilement par en bas.

Pour faciiter la circulation dans les courbes, les crochets de traction peuvent se déplacer latéralement dans les guides des traverses de tête. Les essizux-portcurs sont du type "bissel " avec rappel par ressort. Lcs pivots des essieux bissels, fixés chacun à une entretoise du châssis, sont réglables dans le sens vertical, pour compenser l'usure inégale des bandages des roues motricss et porteusss.

Essicux et Com.nande individuelle systime Westinghouse. - Chaque cssieu-moteur est actionné par úne paire de moteurs de traction, dont la carcasse commune est fixée rigidement au châssis au-dessus de l'essicu.

Le couple moteur de chacun des arbres des rotors est transmis par le pignon calé à l'une des extré nités de ces arbres, à la roue dentée de l'arbre creux concentrique à l'essieu-moteur correspondant. Cet arbre creux cst supporté par la partie inférieure de la carcasse des stators.

Le couple moteur de l'arbre creux est transmis de chaque côté aux roues motrices par 6 ressorts hélicoïdaux. Ces ressorts sont cncastrés à leurs extrémités dans des colliers fixés, l'un sur l'arbre creux, ou le corps de la roue dentée, l'autre sur le corps de la roue motrice. I.es colliers fixés à l'arbre creux pénètrent dans l'espace compris entre les 6 rayons des roues motrices et dépassent le plan de ces roues el rendent ainsi les ressorts facilement accessibles de l'extérieur. Cette disposition permet à l'essieu de prendre une position oblique.

Pour remplacer un ressort d'entraînement défectueux, on l'enlève avec ses colliers pour le remplacer par un jeu de réserve, opération, facilitée par ce fait que les trous de fixation des colliers sont percés suivant un gabarit.

L'encastrement des ressorts dans les deux colliers s'exécute sur un chevalet construit ad hoc.

Les roues motrices n'ont que 6 rayons très résistants, afin de laisser une place suffisante pour les ressorts d'entraînement. Les fusées ont $175 \mathrm{~mm}$ de diamètre et $320 \mathrm{~mm}$. de longueur.

Les essieux-porteurs sont avec disposition de rappel à ressorts.

Les ressorls de suspension des essieux moteurs sont disposés au-dessus des boîtes d'essieux et réunis par des balanciers. Le chàssis principal est supporté par un pivot sphérique réglable.

Plate-forme. - La plate-forme de la locomotive est rivée au châssis. Le tout constitue un châssis solide et rigide. Dans les parois longiludinales, au-dessus de chaque moteur de traction, sont disposés deux grands couvercles amovibles pour l'accès aux collecteurs.

La partie supérieure de la plate-forme est renforcée par des fers à $U$ qui supportent les différents appareils électriques. Sous cette partie supérieure de la plate-forme se trouvt le canal de ventilation pour le refroidissement des moteurs de traction et du transformateur.

Le canal de ventilation est relié aux moteurs de traction par des soufflets en cuir. De grands couvercles sont aménagés sur la partie supérieure de la plate-forme, du côté des collecteurs, pour faciliter le contrôle de ces organes.

Freins el installations à air comprimé. -- Ces locomotives ne possèdent que des freins mécaniques :

10 Freins à main dans chaque cabine, qui, au moyen d'une vis actionnent 6 sabots, la moitié du nombre que comportent les essieux-moteurs.

$2^{\circ}$ Frein double Westinghouse, qui permet de freiner $90 \%$ du poids adhérant.

L'air comprimé servant à actionner les freins ainsı que tous les autres appareils pneumatiques est fourni par un compresseur rotatif à deux phases.

Pour permettre de lever l'un des pantographes, quand l'air comprimé fait défaut, une pompe à main se troure dans la cabine avant, et permet d'obvier ainsi à ce défaut.

Tous les apparils électriques les plus importants (freins, sifflets, sablières, etc.), fonctionnant à air comprimé se manouvrent da chaque cabin?.

\section{Partie Electrique}

Circuits principaux : a) Circul à haute tension (fig. 2). - Le courant principal passe de la ligne de contact Lc par les deux pantographes $\mathrm{P}$, les sectionneurs $\mathrm{S}$, la spire inductive $\mathrm{Bi}$, la tra-

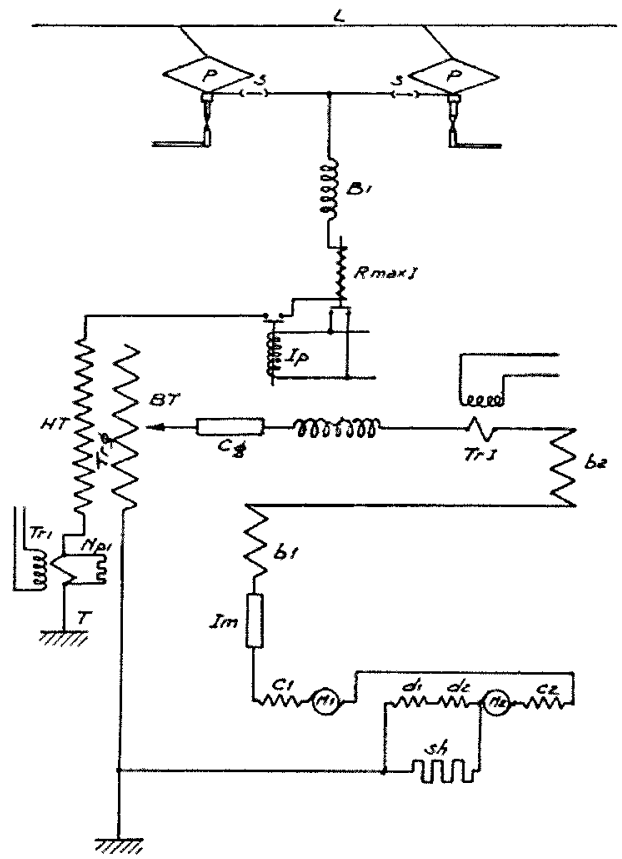

Fig. 2.- Schéma d'une locomotive $A_{e}$ 3/5 
versée dentrée à hante tension, le relais à intensité maximum, $\mathrm{R}_{\mathrm{mxx} \text { I }}$ linterrupteur pruncipal dans l'hule $\mathrm{I}_{\mathrm{p}}$, lenroulement à haute tension du tansformateur à gradins Trg, to transformateur dintensite $T_{1 i}$ muni d'une résistance de prolection c pe el ahoule finalement a harbe de mise a la lerre boulonné an chassis de la locomolive el de hit an rais.

Les deus seclionneurs $S$ peurent blre commandés indépendamment de chaque cabine en isolant ansi les pantographes respectifs. Le fonclionmenent pmemalique des pantographes est aussi aulonome.

La spire induclive $B_{i}$ est, en fail, mo limiteur de tonsion, on cas de surtensions acoidentolles.

Le fonctionnement de linterrupleur prencipal est assujelti à tout un systeme de verrounlage prevu, de fas on a eviter tout accident dinaltention.

La manowre dinchehencht ou de dédenchement de linterrupteur s: fait par command, a histance dictro-pnevmaticue, mais en cas d'urgence, de peut dtre faite à la main.

Son fonctionnement est automatique lorsque lis bomnes conditions de fonctionnement l'exigent. Il tutre en action al declench" pour des surcharges on court-cireuils, lant daus to circuil i hate lension que dans celui des moteurs, comme aussi forsque es mèmes inconvénients se produisent dans les crreuils de chauffage.

Le transformaleur à gradins réduil la lension de la ligne de $15.000 \mathrm{~V}$. dans l'enrouiement P-I à $1.200 \mathrm{~V}$. contre le's extrémilés de co dernier, mais des prises d' courant sur cel enrolilement permettent de ne prélever pour le couraul de traction qu'une tension progressivement variable el sadaplant aux conditions de fonclionnement des moteurs.

La différence de tension entre deux louches de marche consécutives est denviron 50 $\mathrm{V}$.

Sur les dix prises, la tension da moteur de traction pent varier de 0 à $880 \mathrm{~V}$ et cula, gruce à $18^{\prime \prime}$ conlacteurs à graduation, d'une facon très progressite en evilant lous les à-coups possibles.

Les contacleurs de gradualion Cg sonl groupés en 2 balleries et actionnés électro-pneumatiquement des cabines de mécanicien au moyen des combinateurs de marche à asservissement.

Les dix prises de lemronlement à basse lension soni invariablement liées aux diférents contacteurs des deux groupes qui constituent l'ensemble de régulation. Chaque contacteur est pourvu d'un cylindre à air, donl la soupape, combinée avec un électro-aimant, est commandéc par les combinateurs d'asservissement. I.es contacteurs sont munis de soufl.turs magnéliques. Le courant dasservissement est du courant conlinu de 36 à $45 \mathrm{~V}$.

Dès que les conlacteurs indiqués par le combimateur sont enclenchés, la tension correspondante est transmise aux molcurs de traction qui sonl, par groupe, de deux en séric.

Le courant de traction provenant du transformaleur passe done par les contacteurs qui le règlent el qui le dirbsent dans les bobines de réaclance Br (car celles tei sont mises en circuit par les contacteurs auxquels olles sont invariablement liees). Ces trois bobines de réactances sont convenablement insérées dans le circuil de traction, elles servent d'une part, à diviser les tensions captées au transformateur à gradins, el d'autro part à eviler une interruption lors du passage d'une louche sur une autre. La combinaison éludiée de l'inserlion de ces bobines ot commandée par le combinateur d'asservissement rend, au milieu de la bohine intermédiaire, la tension égale à la moyenne arithmétique des tonsions transmises par les conlacteurs aux commenciments des enroulements des bobines extrêmes. b) Circuil de tration. - - Dans le circuil de tractión est inséré un transformaleur d'intensité 'i,r qui fournit le courant poun les appareils de mesure el de controle: Avant d'arriver an moleur, to courant est sommis au controle de l'inverseur de marche Im.

I es inverseurs de marche, a cylindres de commulation, ave doigts de conlad, sonl construits pour etre commandés élofro. pneumatiquement. 1ls peuvent, d'ailleurs, fre lous les deux (dans chaque cabine) commandés à la main, an moy'n d'un poignée spéciale, celle possibifilo el l'éventurilite d'erreurs de commande exigent un verrouillagi parfail, presentant tomb sécurité, ce qui a été oblemu de la fa on la plus salisfaisante

Par linverseur de marche, on peut ehanger le sens de rolation des moleurs de traction en inversant le courant dans les enroplements d'excitation, on bien metre les moleurs de traction hors circuil.

Comme il a été dil plus haul, chaque locomolive a 6 moleurs qui actionnent par groupe de deux chaque essicu motenr. Chaque paire de moleurs est completement enferméc dans une carcasse commune d, au point de vue électrique, ils sont montés en série, landis que les trois paires de moleuss sont comples en parallele.

I.es moteurs de traction monophasé ol a collecteur sont pourvus de trois enroulements d'excilation différents : enroulemenl séric $b$ ( 4 ui se trouvent avant l'inverseu de marche $I_{m}$ ). lenroulement de compensation $C$. en série avec le rolor $\mathrm{N}$ : l'enroulement des pôles auxiliaires en dérivation sur le shun olimique $S_{n}$; de la, le couranl principal est mis a la lere.

Jes enroulements de compensation el des pôles auxiliairs sont destinés à réaliser une commulation pratiquement exemple d'étincelles au collecteur, à loules le's vilesses.

Il n'y a pas de résistance intercalée entre l'enroulement du rotor et les lamelles du collecteur.

\section{Circuits Secondaires}

I a prise de courant pour le chauflage se fail sur l'enroulement basse tension du transformateur.

Au moyen de contacleurs commandés à distance dectropneumaliquement, on peut fournir le couranl de chauflage sous trois lensions diférentes: $600,800,1.000$ volts; un verrouillage spécial, appliqué à ces contacteurs, ne rend possible qu'une scule de ces trois tensions.

Tous les circuits des organis auxiliaires sont alimentes par du courant a 220 volts, tension prise sur te transformatem a gradins.

Tous les moleurs des ventilateurs sont couplés en parallide entre eux et ne peuvent être mis an mạrhe que simullanément.

Le moleur du compresseur est aceomplé directement à bil compresseur rolatif fournissant l'air comprimé nécessaire aux freins el aux apparils pnematiques.

le moleur du groupe molenr-générateur est réuni dans une carcasse commune avec le généraleur de courant continu de 45 volls.

Les circuits d'éclairage sont alimentés par du courant contim à 36 volts, soil directement par les batlerics d'accumulaturs, soil par lo générateur en passanl par une résistance additionnelle qui réduil la lension de 45 à 36 volls.

Le groupe générateur charge deux balleries d'accumulalurs fui, lorsque la locomolive ne se trouve pas sous leusion (part exemple quand elle ne se trouve pas sous la ligne de conlacl) doivent être capables' d'alimenter pendant un certain temps 
Ies circuits d'éclairage et d asservissement. Ces derniers sont en ligne générale, les suivantes:

a) Verrouillage el circuits divers de commande de l'interruptrul principà ;

b) Commandes el verrouillage des inverseurs de marche;

c) Séseau de commande des contacteurs de graduation;

d) Commande de chaufage de train.

\section{Série $A_{e} \quad 3 / 6$}

Dans la description de ce type de locomolives, nous ne nous ulendrons que sur les particularités mécaniques et électriques

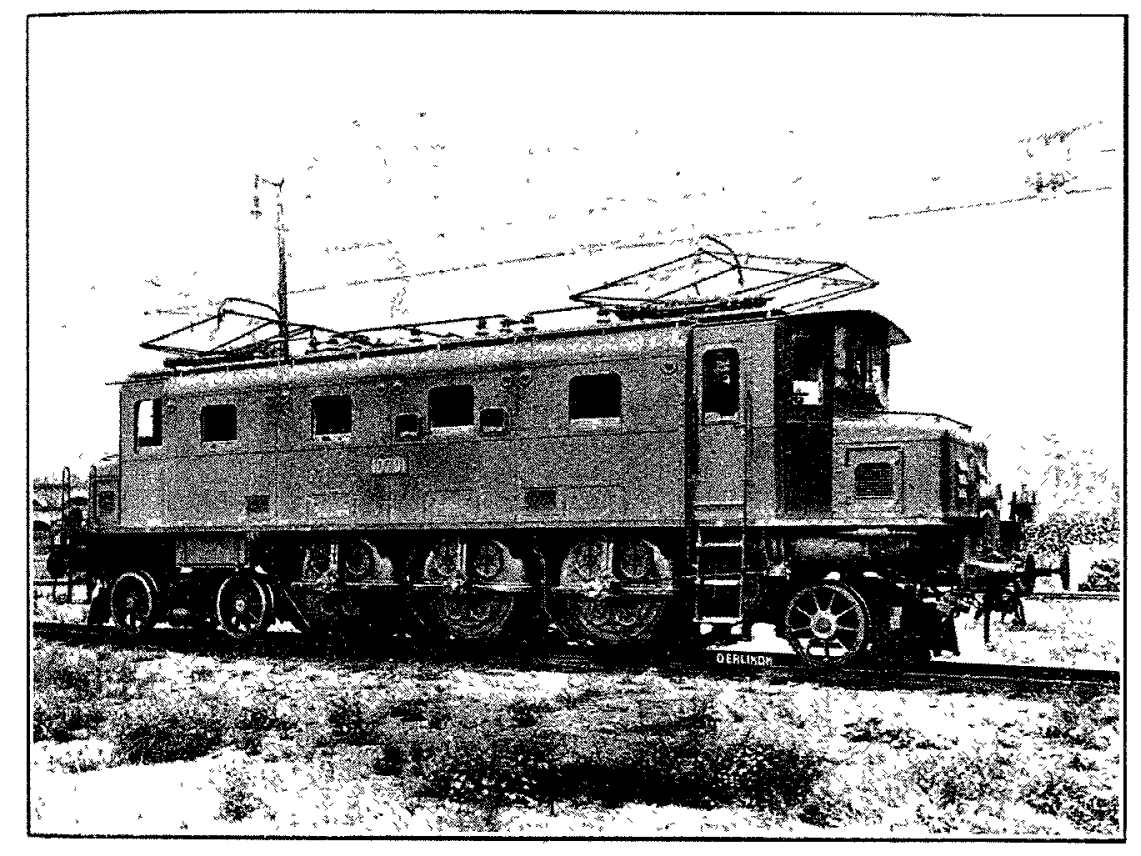

Fig. 3.- Locomotive série $A_{0} 3 / 6$
Puissance unihoraire à la jante pour une vilesse

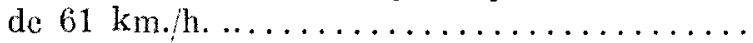

Puissance continue à la jante pour $65 \mathrm{~km} . \mathrm{h} \ldots$

Vilesse maximum ..................

$1.890 \mathrm{CV}$

$1.650 \mathrm{CV}$

$90 \mathrm{~km} / \mathrm{h}$

La dissymétrie de cette locomolive esl nécessitée par la disposilion des appareils électriques et par la répartition du poids

L'essieu porteur, système bissel se trouve à l'arrière. A l'avant se trouve un bogie à deux essieux porteurs, qui porte la crapaudine du pivol sphérique, dont le jeu esl de $80 \mathrm{~mm}$. de chaque côlé. Le pivol et la crapaudine sont en fonte d'acier, et la plaque de glisscment en bronze. Ie rappel, au centre, est effectué par des ressorts à lames et en volules; disposés à l'extérieur du châssis et bien accessibles.

Pour amortir les fortes oscillations latérales de la locomolive, des supports sont fixés aux longerons du bogie, à $5 \mathrm{~mm}$. d'intervalle des appuis correspondants fixés aux longerons du châssis principal.

Commande individuelle B. B. C. - Les trois essieux moleurs son là commande individuelle, système B. B. C.., el chacun d'eux est actionné individucllement par un moteur de traction fixé rigidemenl au châssis, au-dessus de l'essieu correspondant (fig. 4). Le couple moteur de l'arbre du rotor est transmis par un engrenage simple, avec pignon à couronne élastique (rapport de réduction $1,2,57$ ), à la roue motrice correspondanls. Ce tourillon est fixé à un support en fonte dacier.

La roue dentée et la roue molrice corrcspondantes portent chacune deux tourillons reliés par deux bielles et deux leviers à segments dentés; ce mécanisme a pour rôle d'empécher que la transmission de l effort moteur soit influencée par le jeu des ressorts de suspension. Les bielles sont pourvues d'articulations sphériques, permettant à la roue molrice de prendre une position oblique ou de se déplacer laléralement

qui different totalement des parties correspondantes du lỵe précédemment décrit (fig. 3).

\section{Partie Mécanigue}

Caralérisliques gén rale's. - Celte locomotive est destinée au service des trains directs el omnibus, el doit pouvoir remorquer une charge (poids de la locomotive non compris) de :

480 lonnes sur rampe de $10 \%$ à $65 \mathrm{~km} . / \mathrm{h}$; celle mème charge sera transportée à $90 \mathrm{~km}$./h. si la rampe est de $2 \%$

Diamètres des roues nolrices .........

$$
\text { -. porteuses ........ }
$$

$1.610 \mathrm{~mm}$.

Empaltement fixe...............

4.000

Poids en ordre de marche .......... 92,3 tonnes

- adhérent ............. 55,3 -

Nombre de moleurs de traction ....... 3 . 3

Rapport de réduclion dis engrenages... 1/2,57 -

Courant de Lraction allernatif monophasé $15.000 \mathrm{~V}$. et 16 $2 / 3$ périodes.

Foree de traction maximum au démarrage à la

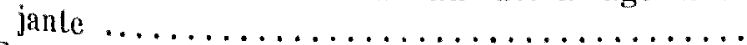

Force de traction unihorairs ì la jante pour une

vilesse de $61 \mathrm{k} . \mathrm{n} . / \mathrm{h} . \ldots \ldots \ldots \ldots \ldots \ldots \ldots$

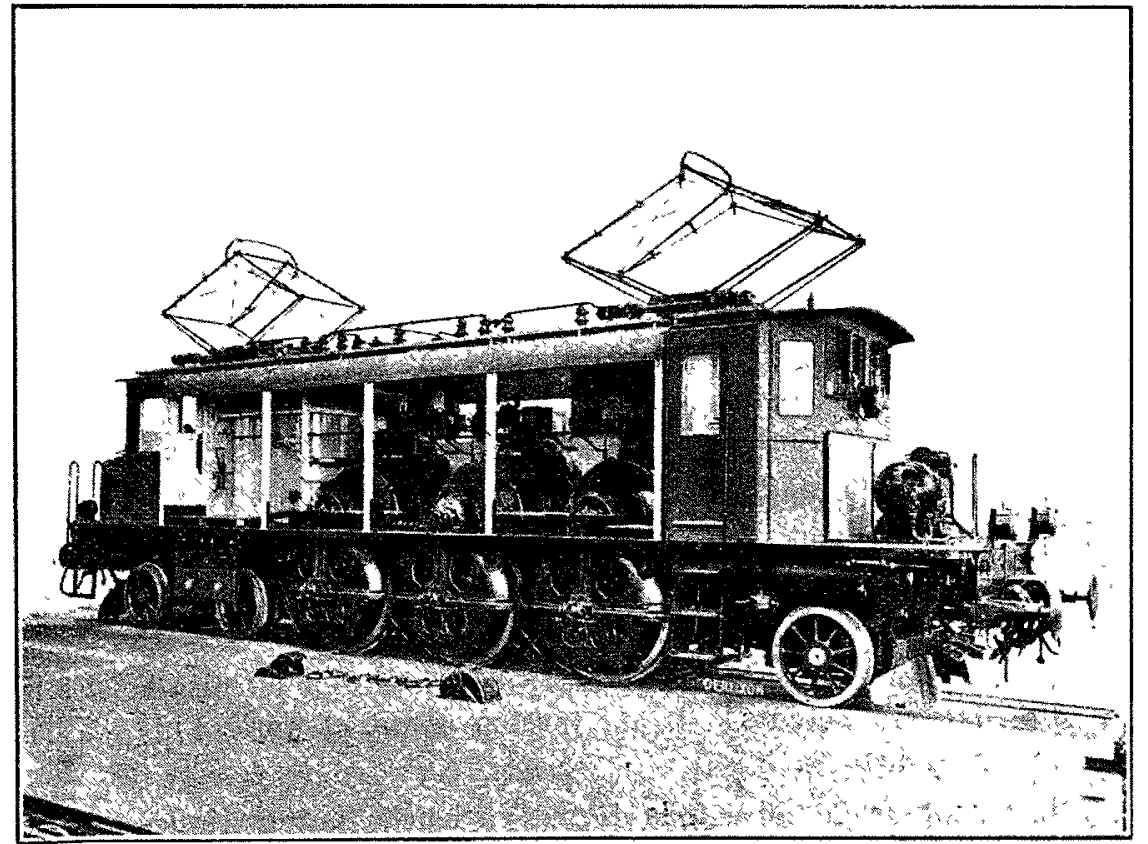

Fig. 4.- Locomotive série $A_{c} 3 / t$, panneaux enlevés (Pholo Oerlikon) par rapport au châssis. L axe de la roue dentée molrice se $14.000 \mathrm{Kg}$. trouve normalement à $25 \mathrm{~mm}$. au-dessus de l'axe de lessien moleur, ceci afin d'obtenir un plus grand rapport de réduc$8.350 \mathrm{Kg}$. Lion. Les organes de transmission sont disposés seulement du 
còté gauche ; il n'y a donc pas ici dellorts alternatifs, comme avec les commandes par biclles, car c'est un couple moteur qui agit. Cette disposition unilatérale augmente le poids de la partic mécanique du cóté gauche : pour équilibrer la charge autant que possible, une partie des appareils électriques et accsssoires a été disposée du côté droit.

Le graissage du mécanisme de transmission est assuré par une pompe à huile adaptée au palier de la roue dentée motrice et actionnée par celle-ci.

Les supports des roues dentées, ainsi que les enveloppes de protection de leur partie inférieure, sont fermés hərmétiquement de tous les côtés, afin d'empècher la pénétration des poussières et les fuites d'huiles. Les roues motrices du côté du mécanisme de transmission sont à plateaux rapportés ou à toile velnue de fonderie, entre les rayons, afin de ne pas faire tourbiilonner la poussière.

Pour faciliter le passage dans les courbes, l'essieu noteur intermédiaire a un jeu latéral de $10 \mathrm{~mm}$. de chaque còté.

Chrissis. - Le châssis est constitué par deux longerons de $25 \mathrm{~mm}$. d'épaisseur, reliés par les traverses de tête et par des entretoises intermédiaires en tôle. Sur le côté extérieur gauche du châssis, sont fixés les gros supports en fonte dacier pour les engrenages et les arbres des rotors; ces pièces contribuent à renforcer considérablement le châssis.

Les crochets de traction peuvent se déplacer latéralement dans les guides des traverses de tête, afin de faciliter la circulation dans les courbes.

La locomotive repose sur quatre points. Les ressorts de suspension des essieux moteurs sont disposés au dessous dis boites d'essicux. Les ressorts des premier et deuxième essieux moteurs d'une part, et ceux du troisième essieu moteur et de l'essieu bissel $d$ autre part, sont réunis par des balanciers.

Ce type de locomotive possède, en outre, des deux freins décrits pour le type précédent, un frein automatique Westinghouse du bogie actionnant 4 sabots. Les cylindres à frein à double piston agissent directement sur les bielles de suspension des sabots.

\section{Partie Electrique}

Circuits principaux. - Cirruits haute tension. - Dans un type de locomotive, les circuits électriques principaux diffèrent du type précédent, et surtout dans le circuit de traction (fig. 5).

Dans le circuit haute tension, on peut signaler un interrupteur de mise à la terre $\mathbf{I}_{\mathbf{T}}$ auquel aboutissent les deux dérivations prises en arrière des sectionneurs $S$, un relais de courant maximum $R_{\max }$, l'interrupteur principal dans l'huile $I p$ avec son relai, un transformateur de courant $T_{\mathrm{r} \mathfrak{i}}$.

L'enroulement haute tension du transformateur principal $T_{p}$ n'est pas unique, il comporte plusieurs prises et on y prélève directement le courant de chauffage.

L'interrupteur de mise à la terre $I_{T}$ sert à établir la mise à la terre des conducteurs à haute tension avant et après l'interrupteur principal.

L'interrupteur de mise à la terre s'enclenche automatiquement chaque fois que l'on ouvre le panneau grillagé du caisson à haute tension; par ce mème fait, les archets ne peuvent plu; être levés tant que le caisson à haute tension est ouvert.

Circuit de traction. - C'est surtout le principe du réglage de la tension aux bornes des moteurs de traction qui est ici complètement différent.
De l'enroulement à tension inférieure du transformateur à gradins partent 18 prises aboutissant à des plots de contact $p$ sur lesquels vont s'appuyer les balais $b$ du graduateur, en captant une tension qui peut varier de 0 à 656 volts pourle réglage de la tension des moteurs de traction (fig. 5).

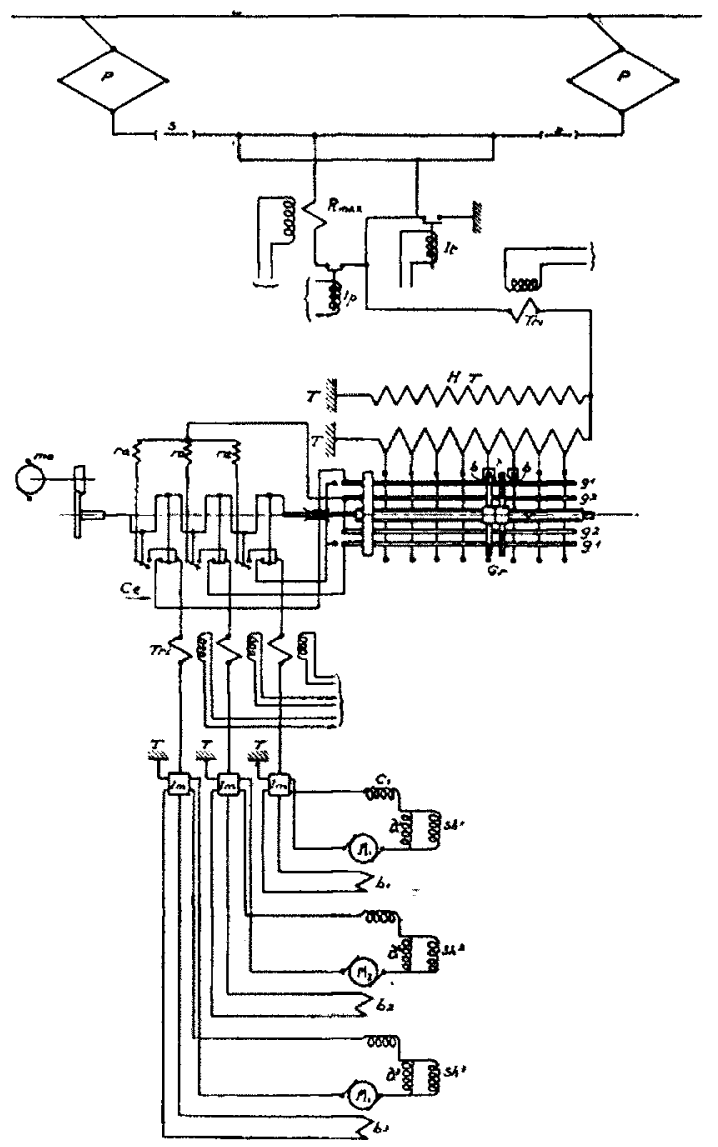

Fig. 5.- Schéma de principe des locomotives série $A_{e} 3 / 6$

Suivant la graduation de ces tensions, on fait varier le couple et le nombre de tours des moteurs de traction et, par conséquent, la puissance et la vitesse de la locomotive.

Du graduateur $G_{r}$, le courant passe par les interrupteurs coupe-étincelles $C_{e}$, les transformateurs d'intensité $T_{n} I$ et aboutit aux inverseurs de marche $I_{m}$, au moven desquels on peut changer le sens du courant dans les enroulements d'excitation, en chan geant ainsi le sens de rotation des moteurs. Les inverseurs de marche sont verrouillés mécaniquemènt avec les combinateurs d'asservissement, de façon à ne pouvoir être manœuvrés que lorsque ces derniers sont dans la position "zéro".

Des inverseurs de marche $I_{m}$, le courant passe par les enroulements principaux série $b$ des moteurs, revient en $I_{m}$ pour se diriger de là, vers les enroulements de compensation C, puis vers ceux $a$ des pôles auxiliaires, qui sont en parallèle avec un shunt thermique $S h$, finalement le courant principal traverse les induits $\mathrm{M}$ des moteurs de traction, revient en $I_{m}$ et, de la, à la masse.

Les trois moteurs sont en parallèle sur la tension qu'établit le graduateur.

Les moteurs de traction sont monophasés à collecteurs, leur couple est transmis aux essieux moteurs par engrenages a jantes élastiques; ils sont refroidis par des ventilateurs.

Dans de nouvelles locomotives, les enroulements de compensation ont été supprimés, mais la teńsion de fonctionnement a été réduite. 
Graduateur contacteur. -- Le graduateur est constitué par deux glissières doubles $f$ et $g_{2}$ sur lesquell s coulissent deux balais, un balai princip $1 b$, et un balai auxiliaire $b$ '. Ces balais sont actionnés au moyen d'une longue vis filetée qui fait tourner un moteur spécial d'entraînement Mr, mais qui peut être le cas échéant, manceuvré à la main.

En position normale de marche, le balai principal se trouve sur une touche de contact, et le balai auxiliaire au milieu, entre cette louche et la suivante. Les interrupteurs coupe-étincclles sont manouvrés par le même arbre que les balais de contact, de facon à coordonner les mouvements comme suit : En position normale de marche, les interrupteurs coupe-étincelles du balai principal sont enclenchés, ceux du balai auxiliaire déclenché.

Pour passer à la touche suivante, les deux balais se meuvent simultanément; en premier lieu, le balai auxiliaire entre en conlact avec la deuxième touche et, en même temps, les interruptcurs coupe-étincelles correspondants s'enclenchent.

Avant que le balai principal abandonne la première touche sur laqnelle il se trouve encore, les interrupteurs coupe-étincolles correspondants se déclenchent; le courant entier passe alors par les résistances d'amortissement et le balai auxiliaire de sorte que le balai yrincipal est completement hors circuil au moment de quitter la première touche. En continuant son mouvement, le balai principal arrive sur la deuxième touche sur laquclle se trouve encore le balai auxiliaire; les interrupteurs coupe-étincelles correspondant au balai principal s'enclenchent de nouveau; donc, au moment où les deux balais sont chacun à moitié sur cette deuxième touche, tous les interrupteurs coupe-étincelles sont enclenchés. Avant que le balai auxiliaire abandonne la deuxième touche, les interrupteurs coupe-étincelles correspondants se déclenchent, de sorte que le balai auxiliaire est hors circuit au moment de quitter la touche. Ic courant entier passe alors par le balai principal.

Entre les glissières auxiliaires $\mathrm{G}_{2}$ et les interrupteurs coupeétincelles sont insérées des résistances d'amortissement $r a$. CEs résistances amortissent les à-coups de courant dans les bobines du transformateur à gradins, mises en court-circuit au moment de leur connexion ou déconnexion avec le graduateur.

Le moteur d'entraînement du graduateur principal Gr est un moteur série à courant continu qui actionne par l'entremise d'un renvoi et d'un accouplement à friction, la tige filetée d'un combinateur auxiliaire d'asservissement du moteur d'entraînement; cetle dernière transmet, à son tour, le mouvement par des engrenages à l'arbre du graduateur principal Gr. Une roue dentée à jante élastique de l'arbre de graduation est munie d'un verrouillage mécanique, empèchant le tourillon d'entraînement de s'engager intempestivement, tant que les balais du graduateur et du combinateur auxiliaire d'asservissement ne sont pas dans leurs bonnes positions.

Le moleur d'entraînement du graduateur est asservi par le combinateur principal placé sous la conduite du mécanicien, ainsi que par le graduateur auxiliaire. Il est commuté automatiquement sur marche avant ou arrière au moyen d'un relai polarisé. A chacune des 18 positions du combinateur d'asservissement correspond une mise en circuit d'asservissement telle qu'elle provoque la marche du moteur d'entraînement dans un sens déterminé et imposé par un relais, tant que le grarluateur principal ne s'ast pas placé dans la position voulue p:r le régime de marche $t$ t indiqué par le combinaleur commandé par le mécanicien

\section{Série $C_{e}$ 4/6 - Automotrices à commande directe et multiple à distance}

Généralitos. - Les automotrices sont destinées essentiellement au service des trains de banlieut et des trains-tramways. Afin de Fouvoir réduire la durée des parcours et obtenir une vitesse moyenne élevée, ccs automotrices sont munies de motenrs permettant d'obtenir une trés grande accélération.

La vitesse maximum que doit fournir l'automotrice lorsqu'elle est utilisée comme véhicule moteur est de $75 \mathrm{~km} . / \mathrm{h}$; en pente à courant déclenché, elle peut atteindre $90 \mathrm{~km}$. h.

L'application du dispositif de commande multiple à distance permet d'utiliser les automotrices pour le service des trainsnaveltc. Ces trains sont actionnés soit pa: une automotrice en tête du train, de véhicules à conduites d'asservissement éltctrique et pneumatique et d'un wagon de commande $t \mathrm{n}$ queue ou, encore, d'une automotrice à chaque extrémité du train et de véhicules à conduites d'as'sivissement électrique et pneumatique, intercalés entre ellts.

Les caractéristiques principales de ces locomotives sont :

Poids en ordre de marche ........... 85,3 tonnes

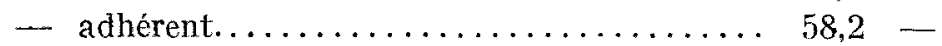

Diamètre des roues motrices........... $1.040 \mathrm{~mm}$.

porteuses $\ldots \ldots \ldots \ldots \ldots \ldots . \quad 850 \quad$

Empattement total ................ 10.000 entre les essieux moteurs d'un bogle. 3.200 -

Force de traction unihoraire à la jante à $48 \mathrm{~km} . / \mathrm{h} \ldots \quad 4.360 \mathrm{~kg}$. - maximum au démarrage ...... 9.000 -

Puissance unihoraire à la jante à $48 \mathrm{~km} . / \mathrm{h} . . . .780 \mathrm{Ch}$. continue à $54 \mathrm{~km} . / \mathrm{h} . \ldots \ldots \ldots \ldots \ldots 640 \ldots$

\section{Partie Mécanique}

Vu le poids considérable de l'équipement électrique, les automotrices sont munies de deux bogies à trois essieux, dont les deux extrêmes sont des essieux moteurs et celui intermédiaire est un essieu porteur.

Les bogies supportent le châssis et la caisse de l'automotrice. Les appareils électriques, transformateur, asservissement, compresseur, inverseurs sont fixés au châssis sous la caisse de l'automotrice et ne se retirent que par le bas.

Le châssis est constitué par des longerons en fer profilé, entretoisés et consolidés par de fortes armatures.

Les appareils combinés de choc et de traction aux deux extrémités du châssis sont du type P.-L.-M. à compensation.

Le châssis repose au milieu de chacune des bogies sur un gros pivot sphérique porté par un balancier transversal.

Les bogies sont constitués par de robustes châssis en tòle, entretoisés et renforcés. La suspension du châssis du bogie, sur les trois essieux, comporte quatre jeux de trois ressorts hélicoïdaux concentriques.

Chaque essieu moteur est actionnć par un moleur électrique reposant d'une part, sur lessieu, et suspendu élastiquement; d'autre part, au châssis (suspension de tramway).

Le couple moteur est transmis à l'essieu par un engrenage dont la grande roue est à jante élastique.

Le rapport de réduction entre le moteur et l'essicu est de 1/3,65. Les engrenages, avec graissage à huilc, sont enfermés dans une enveloppe de protection en aluminium, en deux parties. 
Lautomotrice posside les freins suivants, à deux sabots sur charge rour :

10 Frein a main, dans chaque cabine, agissant sur le bogie correspondant:

20 lirein aubonatique Westinghouse à action rapide;

30 Frein moiderable Westinghouse.

Le reste de l'installation ressemble dans se's lignes générales à tous les autres lypes de locomotives ou locomotrices.

\section{Partie Electrigue}

Circuls principaux. - Ce type de locomolrice est, an point de vue électrique, très semblable a la parlie électrique du lype Ae $3 \overline{3}$.

I a seule différence de principe réside dans la possibilité de commande à distance el de lype mulliple. A cel effel, les circuits dasservissement sont parliculièrement éludiés en regroupant dans un accouplement spécial les fils de commande de la marche aulomalique.

a) Cirut hate tension. - Dans le circuit haute tension, nous retrouvons comme d habilude, en partant de la ligne de contact L (fig. 6).

I.es pantographes $\mathrm{P}$, les seclionneurs $\mathrm{S}$, la spirale induclive $B_{\mathbf{i}}$

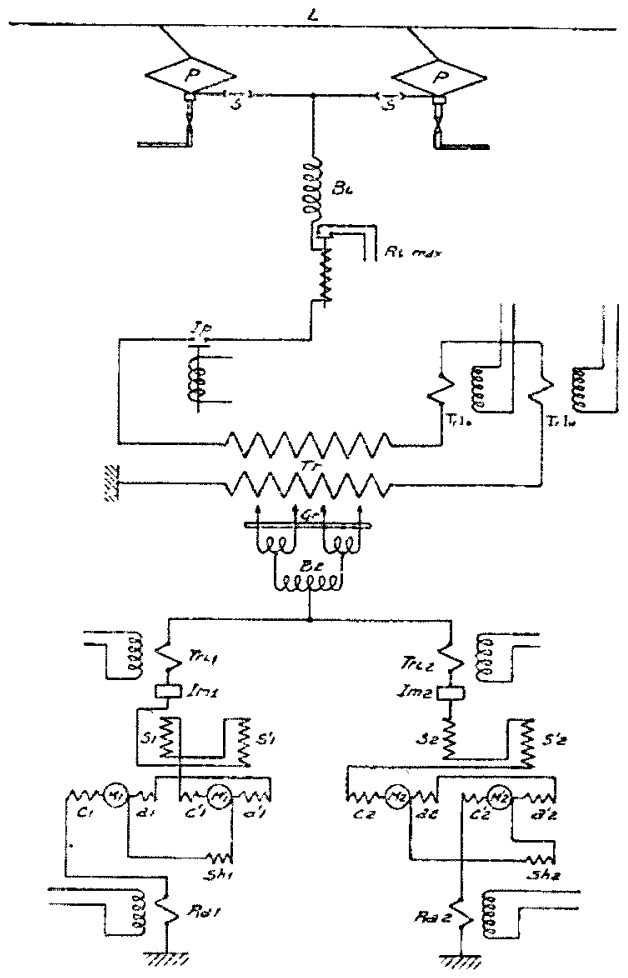

Fig 6. - Schéma de principe des locomotives série $C_{8} 4 / 6$

(protection contro los surtensions évenluelles), le relais à intensilé maximum $\mathbf{P}_{\mathbf{i} \text { max }}$, l'interupteur principal dans l'hnile $I_{p}$ avec une résislance de protection destinée à amortir les à-coups de courant, lors de 1 enclenchement du transformateur à gradin et, finalement, on aboulit à l'enroulement haute tension du transformaleur $T_{r}$ qui réduit la tension de 15.000 volts aux différentes tensions nécessaires pour lalimentation des moleurs de traction et du chauffage du train.

Le circuil principal se conlinue en passant par deux transformateurs d'intensité du courant principal : Trla pour les instruments de mesure de l'autonotrice el ' $\Gamma_{r \text { Iw }}$ pour les instruments de mesure du wagon de commande; enfin, il aboutil à l'enroulement basse tension du transformatcur et, de là, à la barre de mise à la terre. b) Circuit de traction. - - Le circuit de traclion n'est caracte. risć que par co fait, qüil y a quatre moteurs accouples dectriquement pay groupes de deux en série, el les deux grotipes en dérivalion sur la lension mise à disposition par le contacteu du graduation. Ici aussi, comme dans le type $A_{e} 3 / 6$, c'est. un graduateur par contacteurs commandés électropneumaliquomenl, commo nous lavons vu. les combinateurs d'asservisse. ment indiquent el commandent l'enclenchement des soupapes des contacteurs qui délerminent la Lension de scrvice. Le courant de traction ainsi élabli, traverse les bohines de réactance $33_{\text {, }}$ que nous avons mentionnées dejà dans le premier lype $A_{\mathrm{c}} 3 / 5$.

De là, le courant de traction se divise en deux parties se dirigeant dans chayue branche de deux groupes de moteurs. Il traverse lis transformaleurs d'intensité $T_{r j}$, les inverseurs de marche Im el, de là, les moleurs de traction, en parcourant succ ssivement les enroulements d'excilation en série 3 , les rolors .1 , les enroulements des pôles auxiliaires a couplés én parallele avec un shunt thermique $\mathrm{S} h$, les enroulements de compensation 'r et de là, par le relais d'accélération l'a, la barre de mise à la terre.

I.e courant d'asservissement est toujours le courant continu à $36-45$ volls. I.es conlacteurs, munis de souflage magnétique, sont actionnés à l'air comprimé à 5-7 atm.

Combinateur d'asservissement. - La parlicularité la plus saillante de ces automotrices réside dans la possibilité de commande aulomatique des combinateurs. Les combinateurs d'asservissement, aclionnés par le mécanicien, sont munis dü accouplement à ressort en spirale intercalé entre la manivellé el le cylindre du combinateur. Celle disposition permel, lorsqu'on utilise la commande automatique (ou à distance), de placer la manivelle du combinateur d'asservissement direct. ment sur la touch' de marche correspondant à la vitesse désirée, Il faul pour cela disposer la poignée du commulateur de commande, qui se. trouve sur la table, dans la position " automalique ".

Alors, en lournant la manivelle du combinaleur, te ressort en spirale se tend, el le circuit du courant d'asservissement de la commande automatique se ferme. Un électro-aimanl, verrouillant le cylindre du combinateur d'asservissement, libère un instant ce cylindre qui, entraîné par le ressort en spirale, avance alors d'un cran. Un cliquet d'arret maintient un instant le cylindre dans cette position, puis le libère à nouveau. El ainsi de suite jusqu'à ce que le cylindre du combinateur, toujours entraîné par le ressort, ait passé successivement lous lés crans el soit arrivé à celui correspondant à la position de la manivelle du combinateur. Dans cette position, le circuil dı courant d'asservissement de la commande automatique s'ouvre et le dispositif de vertouillage immobilise le combinateur jusqu'à ce que le mécanicien fasse avancer de nouveau la manvelle.

Pour no pas surcharger les molcurs, le passage d'une touche à l'autre ne doit se faire que quand l'intensilé du courant des moleurs le permel.

Le fonclionnement par commande à distance, privé de la surveiilance direcle du conducteur, doil loulefois atre pourvu de loule garantie de pouvoir s'effectuer dans de bonnes condilions, par un autocontrôle. A cel effet, el prenant comme crilérum le bon fonclionnement des moleurs de.traction, on a installé les relais d'accélération $R_{a}$ comme il a élé indiqué plus laat. Ils ont l'offlce de couper le courant d'asservissement de la commande automalique dès que, par progression sur une louche supérieure du combinateur, l'intensité du courant des moteurs de traction, dépasse la valeur maximum fixée. Le cylindre du 
combinateur reste alors verronillé jusqu'à ce que, en raison de laceleration du train, l'intensité du courant des moteurs de taction soil relombée à la limite admise. A ce moment, It circuil du courant d'asservissement de la commande aulomatipue se ferme de nouveau el libère le cylindre du combinateur diasservissement.

Commande à distance. -... I a commande directe des locomotwes ou automotrices est mème sous la surveillance du conducteur, uniquement el entièrement assurée par le contrôle à dislance: car, en effel, le conducleur ne lait que " donner des ordses en manouvant ic combinateus, ordres que le circuil d'assertissement se charge d'exéculer scrupuleusement. Obtenir la commande à parlir d'unc distance plus grande revient à dendro le réseaul du circuil d'asservissament jusqu'à l'endrout dou la commande doil ètre effectuée, et c'est ce qui est cxéculé au noỵ d'un cable se terminant par une lête daccouplenent de rolle canalisation de la commande multiple.

Dans celle canalisation, se trouvenl les circuils d'asservissement des arehets, de linlerrupleur principal, des inverseurs de marche, des contacteurs de graduation de la commande automatique du conlacteur du compresseur, de la po mpe à huile, du groupe générateur, du contacleur de chauffage à 800 ou 1.000 rolts, ainsi que le circuit du vollmetre du wagon de commande pour la tension de la ligne de contact de d'amperometre mesurant l'intensité du courant principal.

Ce dispositif de commande multiple a distance pernet de conduire le train :

a) Depuis une autonotrice;

b) Depuis un wagon de commande, ou bien d'une automotrice utilisée commo wagon ds commande.

Lorsque la condule du train s'effectue depuis une aulomolrice, le courant d'asservissoment pour la commande mulliple à distance est fourni par le groupe moteur-générateur ou les balteries de l'aulomotrice litulairc on desservie; les batteries de l'automotrice non desservic, n'ont à fournir que le courant de déclenchement des relais de ce véhicule.

Lorsqu: le poste de manceuve se trouve sur un Wagon de commande, le courant d'asservissement pour la commande mulliple a dislance est fourni par le groupe d'éclairage électrique de ce wagon.

(A suivre)

\section{DOCUMENTATION}

\section{L'utilisation de la force des mouvements de la mer par le bélier-siphon maritime à chambre barométrique}

Apres la solution de Georges Claude et Boncherot, sur l'utilisation de l'energic thermique des mers et celle tirant partic die ha chute proluite par les marées, accumulant l'eau dans de grands bassin, une nouvelle due -à M. Paul Grasset est le bélier-siphon maritime à chambre baronetricue.

Elle utilise l'énergie de la houle, énergie extrèmement variable, et dont la puissance peut aller de 1 à 20 et même 30 . L'installation comprencl un réservoir élevé, vide, qui remplit à la fois le rôle d'accumulateur, de régulateur et de distributeur d'eau aux turbines. Deux colones ascendante et descendante aboutissent au réservoir. Elles constituent un siphon.

L'énergie par à-couns des vagues se transforme ainsi cu une énergie continue. Une installation d'essai est en construction dans les falaises du Phare de Biarritz.

La Technique Moderne, $1^{\mathrm{er}}$ auùl 1933.

\section{L'interconnexion bouclée}

Quand un système de feeders, reliant différents centres est bouclé, la valeur de l'énergie totale produite par chaque centre est inlépendante des liaisons et déterminée par les régulateurs des turbines; mais la répartition fle cette énergie entre les différents fceders est fonction de la configuration du réseau.

Cette répartition 'naturelle n'est pas, en général, la plus désirable, et l'on doit recourir à des tensions additionnelles conve- nables, pour obtenir une répartition répondant le micux aux exigences du service.

L'auteur examine les différents cas, répartition naturelle et répartition forcée, ainsi que les divers moyens pour obtenir cette dernière, et les décalages angulaires dans les machinés synchrones couplées en parallèle. Scuence el Industrie, aôt 1933.

\section{L'étude de l'influence du coup de bélier sur le réglage des turbines hydrauliques par la méthode d'Alliévi}

Le reglage automatique des groupes hydro-clectrigues alimentes par une conduite sous pression est souvent influencé. parfois mêne considérablement gêné, par le coup de bélicr.

lors d'une décharge, par exemple, le déséquilibre des couples moteur et résistant donne jieu à une accélération augulaire que le rigulateur cherche a corriger en fernant le vannage. Mais cette ferncture provoque une surpression qui peut compenser, et même at dela, leffet rle diminution de la section de lorifice d'ecoulement. En cas de charge c'est le phinomène inverse cui se produit.

La puissance livrce à la turbine varie donc en sens contraire the la puissance demandfe. Ce n'est qu'après un temps fonction de la longueur de la conduite, que ce phénoméne d'inversion disparait ot qu'un réglage effectif devient possible.

l.e coup de bélicr est un phénomène rythmique. Lorsque la longueur de la conduite devient grande par rapport à la chute, le réglage automatique devient difficile. Les conditions de réglage peuvent être notablement anéliorćes par l'cmploi de dispositifs spéciaux, dont les plus courants sont les orifices compensateurs synchrones, qui supprinent pratiquenent le coup de bélier de fermeture, les tubes piczomctriques dextromite, ou encore les chambres l'air.

Lorsque la conduite est munic, immediatement avant lobturateur, d'un tube pićzométrique vertical, surmonté d'un réservoir avec déversoir, qui y maintient le nivean constant, la surpression maximum est réduite, les lois du coup de belier sont profondement modifićes et les conditions alc réglage sont sensiblement amćliorées.

La chambre d'air supprime toule variation de prossion au dónut de la mancuve, en faisant varier le lobit proportionnellement au degré d'ouverture. Le volume minimum de la chambre peut se calculer en posant les conditions suivantes : $l^{0}$ La charge du coup de bélier ne doit pas dépasser une valeur fixce d'arance; zo l'énergie de l'eau doit toujours etre décroissante; $3^{\circ}$ le point d'inflexion de la courbe $w=f(t)$ dont les ćuations revelent l'existence sera un point de tangente horizontale correspoudant à la fin de la phase directe. M. P.OGYE 